\title{
AVALIAÇÃO DAS PROPRIEDADES HIGROSCÓPICAS DA MADEIRA DE Eucalyptus saligna Sm., EM DIFERENTES CONDIÇÕES DE UMIDADE RELATIVA DO AR ${ }^{1}$
}

\author{
José de Castro Silva² e José Tarcísio da Silva Oliveira²
}

\begin{abstract}
RESUMO - O estudo da higroscopicidade é indispensável para o entendimento da trabalhabilidade, estabilidade dimensional, resistência mecânica e durabilidade natural da madeira. Neste trabalho objetivou-se a avaliação do teor de equilíbrio higroscópico para diversas condições de umidade relativa do ar, bem como da retratibilidade linear e volumétrica e da densidade básica da madeira de Eucalyptus saligna $\mathrm{Sm}$. A madeira utilizada foi proveniente de árvores com 16 anos de idade, procedentes de talhões experimentais da EMBRAPA Florestas de Colombo, Paraná. Amostras com dimensões de 1,0×2,0×3,0 cm, sendo a última na direção longitudinal, foram colocadas em uma câmara fechada, sob ventilação, próximas de recipientes com soluções salinas supersaturadas, a fim de atingir determinada condição preestabelecida de teor de equilíbrio higroscópico. Após o equilíbrio da umidade da madeira nas distintas condições de umidade relativa, as amostras foram secas em estufa, para posterior avaliação. Os dados relativos à umidade de equilíbrio ajustaram-se muito bem às condições de umidade relativa adotadas neste estudo, tendo sido possível estimar com grande precisão o teor de equilíbrio higroscópico, para a faixa de aproximadamente 20 até $100 \%$ de umidade relativa. A madeira em estudo apresentou dados de retratibilidade bastante elevados, se comparados aos de outras da mesma faixa de densidade. Apesar dos elevados coeficientes de contração, o fator anisotrópico ou relação $\mathrm{T} / \mathrm{R}$ mostrou-se próximo daquele encontrado na grande maioria das madeiras comerciais brasileiras. Verificaram-se ainda coeficientes de contrações mais suaves nos teores de umidade abaixo de $17 \%$.
\end{abstract}

Palavras-chave: Propriedades higroscópicas e físicas, Eucaliptus saligna e madeira.

\section{EVALUATION OF THE HYGROSCOPIC PROPERTIES OF Eucalyptus saligna Sm. WOOD UNDER DIFFERENT OF RELATIVE HUMIDITY CONDITONS AIR}

\begin{abstract}
The study of hygroscopic properties is essential for understanding the workability, dimensional stability, mechanical resistance and natural durability of wood. This work aimed to evaluate the hygroscopic balance content of several air relative humidity conditions, transverse and volumetric shrinkage and specific gravity of the Eucalyptus saligna Sm. wood. The wood used originated from Eucalyptus saligna Sm. trees, from the EMBRAPA experimental stands in Colombo, Paraná. The dimensions of the samples were $1.0 \times 2.0 \times 3.0 \mathrm{~cm}$, with the latter in the longitudinal direction. Samples were placed in a closed chamber under ventilation near supersaturated saline solutions until they reached the equilibrium moisture under the chamber conditions. After reaching the equilibrium moisture under several conditions, the samples were oven-dried and weighed. The hygroscopic parameters, shrinkage and specific gravity were finally determined. Data on equilibrium moisture agreed very well under the relative humidity conditions to a range from $20 \%$ to $100 \%$ of air relative humidity. Shrinkage parameters were very high, compared to those of other woods of same density. Despite the high shrinkage coefficients, the anisotropic factor or relation between shrinkage in the tangential direction and that in the radial direction $(T / R)$, were close to the values found for most Brazilian commercial woods.
\end{abstract}

Key words: $\quad$ Physical and hygroscopic properties, Eucalyptus saligna and wood.

1 Recebido para publicação em 18.9.2001.

Aceito para publicação em 19.2.2003.

2 Departamento de Engenharia Florestal da Universidade Federal de Viçosa - UFV, 36571-000 Viçosa-MG. 


\section{INTRODUÇÃO}

Embora a umidade não possa ser considerada como uma característica intrínseca da madeira, o seu estudo é indispensável por se tratar de um parâmetro que afeta o comportamento do material, quanto à trabalhabilidade, estabilidade dimensional, resistência mecânica e durabilidade natural. Por ser um material orgânico e de estrutura complexa e heterogênea, a madeira é altamente higroscópica, retraindo-se e inchando de acordo com a umidade do ambiente. Essa variação no teor de umidade afeta a geometria das peças em serviço, em virtude da retração e do intumescimento, o que afeta as características de resistência mecânica dos elementos estruturais.

O processo de secagem da madeira resulta em contrações lineares diferenciadas, manifestadas nos três planos fundamentais de estudo da madeira, ou seja, nos planos longitudinal, tangencial e radial. Segundo Hillis \& Brown (1978), a utilização da madeira do gênero Eucalyptus, oriunda de árvores jovens e de crescimento acelerado, é mais difícil, por apresentar contrações excessivas que são seguidas pelo aparecimento de defeitos, como rachaduras e empenos.

Em função da elevada anisotropia dimensional, que ocorre na maioria das madeiras de eucalipto, torna-se imprescindível o conhecimento dos mecanismos físicos que regem os fenômenos de retratibilidade. Tal conhecimento servirá de subsídio para a correta condução do processo de secagem e de utilização final, com o intuito de eliminar ou minimizar a ocorrência de possíveis defeitos, comumente encontrados na madeira, assegurando a obtenção de um produto final com qualidade e bom desempenho. Apesar dos elevados coeficientes de contração apresentados por algumas espécies, autores como Barroso (1983), Boland et al. (1984) e Hillis \& Brown (1978) citaram a utilização da madeira de Corymbia citriodora, Eucalyptus grandis e Eucalyptus saligna em assoalhos industriais e residenciais.

As variações dimensionais provocadas pela contração e pelo inchamento da madeira, segundo Durlo \& Marchiori (1992), constituem, conjuntamente com a anisotropia, características indesejáveis da madeira, limitando o seu uso para diversas finalidades ou, ainda, exigindo técnicas específicas de utilização. A contração e o inchamento, em última análise, correspondem às alterações na quantidade de água de impregnação, isto é, a secagem ou o ganho de umidade abaixo do ponto de

R. Árvore, Viçosa-MG, v.27, n.2, p.233-239, 2003 saturação das fibras, ou cerca de $28 \%$ de teor de umidade em base seca. A maior alteração dimensional da madeira se manifesta no sentido tangencial aos anéis de crescimento, seguida pela dimensão radial, sendo praticamente desprezível no sentido longitudinal. Outro importante índice para avaliar a estabilidade dimensional da madeira é o coeficiente ou fator anisotrópico, definido pela relação entre as contrações tangencial e radial (T/R).

Panshin \& De Zeeuw (1980) afirmaram que as variações dimensionais da madeira são desiguais ao longo das três direções estruturais, sendo função de sua característica anisotrópica. Em geral, observa-se que a contração na direção tangencial é aproximadamente duas vezes maior que na direção radial. Os autores afirmaram que a utilização da madeira para obtenção de produtos finais de alta estabilidade requer um fator anisotrópico baixo. Existe uma situação em que a madeira não perde nem absorve água do ambiente, ou seja, a umidade da madeira está em equilíbrio com a umidade relativa do ar, o que é chamado de umidade de equilíbrio da madeira. Ainda segundo os autores, a madeira deverá sempre ser utilizada com uma umidade próxima ao teor de umidade de equilíbrio higroscópico. Uma vez seca a uma condição de umidade de equilíbrio com as condições ambientais, a madeira não irá mais apresentar problemas associados à retratibilidade, como empenamentos e fendilhamentos.

Segundo Oliveira (1998), o conhecimento das propriedades higroscópicas é, sem dúvida, a chave para a utilização bem-sucedida da madeira. Sabe-se que a madeira seca a um teor de umidade igual ou próximo àquele de equilíbrio com as condições de uso da madeira poderá ter seus problemas referentes à umidade minimizados ou definitivamente eliminados.

Segundo Kollman \& Côté (1968), a diferença entre a retratibilidade tangencial e a radial pode ser explicada pela influência restritiva dos raios na direção radial e também pelo arranjo helicoidal diferente das microfibrilas nas paredes tangenciais e radiais. Uma das causas das propriedades anisotrópicas da madeira é a orientação das micelas, fibrilas e fibras que formam o tecido lenhoso. Masseran \& Mariaux (1985) relataram que em grande parte das folhosas as maiores diferenças da retratibilidade tangencial em relação à radial são atribuídas às características anatômicas. Segundo os autores, o volume dos raios, a dimensão radial das fibras e as diferenciações químicas entre as paredes radiais e tangenciais são responsáveis pela anisotropia da madeira. Tsoumis (1991) afirmou que 
a magnitude da contração e da expansão da madeira tornase maior com o aumento da densidade. Hillis \& Brown (1978) relataram que, na Austrália (seu país de origem), a madeira proveniente de árvores de eucalipto que cresceram rapidamente, normalmente, apresenta contração excessiva e, também, defeitos de secagem, como empenamentos e fendilhamentos, que tendem a ser piores em madeiras de menores densidades (abaixo de $650 \mathrm{~kg} / \mathrm{m}^{3}$ ). Lima (1996), estudando madeira de Eucalyptus microcorys, de 20 anos de idade, verificou que a contração volumétrica total e parcial estava positivamente correlacionada com a densidade básica, de forma bastante significativa.

O objetivo deste trabalho foi avaliar o teor de equilíbrio higroscópico para diversas condições de umidade relativa do ar, bem como a retratibilidade linear e volumétrica e a densidade básica desta madeira, nestas condições de umidade de equilíbrio higroscópico.

\section{MATERIAL E MÉTODOS}

A madeira utilizada no ensaio experimental foi proveniente de árvores de Eucalyptus saligna Sm., com 16 anos de idade, procedente de talhões experimentais da EMBRAPA Florestas, de Colombo, Paraná. Os ensaios foram realizados no Laboratório de Propriedades Físicas e Mecânicas da Madeira do Departamento de Engenharia Florestal da Universidade Federal de Viçosa. Utilizaramse cinco árvores, retiradas aleatoriamente das linhas internas do povoamento, aproveitando-se toretes de seções correspondentes ao DAP. Da porção diametral do torete, tomaram-se quatro posições eqüidistantes $(0,33$, 66 e 100\%) no sentido medula-casca, com 20 repetições por posição, totalizando 400 amostras. As amostras de formato retangular apresentaram dimensões de $1,0 \times 2,0 \times 3,0 \mathrm{~cm}$, sendo a última medida no sentido longitudinal. Tais amostras, inicialmente em estado verde, foram colocadas em diferentes condições de umidade relativa, proporcionadas por diferentes soluções salinas supersaturadas (GAL, 1957), conforme o Quadro 1.

As amostras foram colocadas em uma câmara fechada, sob ventilação, próxima de soluções salinas supersaturadas, monitoradas com termohigrógrafo e psicrômetro. A massa das amostras foi monitorada periodicamente até que atingissem massa constante, sendo elas, então, pesadas e tomadas as dimensões lineares e o volume, através do método da balança hidrostática. As soluções foram substituídas assim que as amostras atingiam massa constante para uma determinada condição de umidade relativa, considerando que a utilização seqüencial dos sais foi correspondente à umidade relativa decrescente. Além das soluções salinas, foi utilizada sílica gel, para proporcionar uma condição de umidade relativa mais baixa, devido à não-disponibilidade de um sal que pudesse proporcionar tal condição.

Quadro 1 - Relação das soluções salinas utilizadas e respectivas umidades relativas, à temperatura de $27^{\circ} \mathrm{C}$

Table 1 - Saline solutions and respective relative humidities at $27^{\circ} \mathrm{C}$

\begin{tabular}{|l|l|c|}
\hline \multicolumn{1}{|c|}{ Sais } & \multicolumn{1}{|c|}{ Nomenclatura } & $\begin{array}{c}\text { Umidade } \\
\text { Relativa (\%) }\end{array}$ \\
\hline $\mathrm{ZnSO}{ }_{4} \cdot 7 \mathrm{H}_{2} \mathrm{O}$ & Sulfato de zinco & 95,0 \\
$\mathrm{NaCl}$ & Cloreto de sódio & 74,0 \\
$\mathrm{NaNO}_{2}$ & Nitrito de sódio & 66,0 \\
$\mathrm{Mg}\left(\mathrm{NO}_{3}\right) \cdot 6 \mathrm{H}_{2} \mathrm{O}$ & Nitrato de magnésio & 60,0 \\
$\mathrm{KSCN}_{\mathrm{MgCl}_{2}}$ & Tiocianato de potássio & 50,0 \\
$\mathrm{CH}_{3} \mathrm{COOK}$ & Cloreto de magnésio & 42,0 \\
$\mathrm{SiO}_{2}$ & Acetato de potássio & 33,0 \\
\hline
\end{tabular}

Fonte: Gal (1957).

Finalmente, as amostras foram secas em estufa a $103+2{ }^{\circ} \mathrm{C}$, para tomadas da massa, das dimensões e do volume na condição anidra. Encerrada a coleta dos dados, procederam-se aos cálculos dos parâmetros de higroscopicidade, retratibilidade e densidade, bem como à análise estatística e confecção de gráficos ilustrativos e dos parâmetros estudados.

\section{RESULTADOS E DISCUSSÃO}

Os valores relativos à umidade de equilíbrio higroscópico para diferentes condições de umidade relativa do ar, bem como os valores para retratibilidade e densidade básica para a madeira de Eucalyptus saligna, estão nos Quadros 2 e 3, respectivamente.

Através do Quadro 2, tem-se uma idéia dos valores de umidade a que essa madeira deverá ser seca, em função dos diferentes locais de uso. Os valores revelam um comportamento higroscópico bastante homogêneo, tanto do coeficiente de variação como do desvio-padrão. Oliveira (1998), estudando o comportamento da madeira de Eucalyptus, proveniente de árvores de 16 anos aproximadamente, encontrou diferenças entre as sete espécies estudadas para o teor de equilíbrio higroscópico (TEH), alcançado durante o ensaio de retratibilidade.

R. Árvore, Viçosa-MG, v.27, n.2, p.233-239, 2003 
Quadro 2 - Valores médios* de umidade de equilíbrio (\%) para a madeira de Eucalyptus saligna submetida a diferentes condições de umidade relativa para temperatura de $27^{\circ} \mathrm{C}$

Table 2 - Average of equilibrium moisture values in percentage for Eucalyptus saligna wood submitted to different conditions of relative humidity of the air at $27^{\circ} \mathrm{C}$

\begin{tabular}{|c|c|c|c|c|c|c|c|}
\hline \multicolumn{8}{|c|}{ Umidade Relativa (\%) } \\
\hline 28 & 33 & 42 & 50 & 60 & 66 & 74 & 95 \\
\hline 5,7 & 6,9 & 8,4 & 10,2 & 11,9 & 14,1 & 16,3 & 23,1 \\
$(14,4)(0,8)^{\frac{1}{-}}$ & $(8,9)(0,6)$ & $(7,2)(0,6)$ & $(6,8)(0,7)$ & $(6,1)(0,7)$ & $(4,8)(0,7)$ & $(4,8)(0,8)$ & $(12,7)(2,9)$ \\
\hline
\end{tabular}

* Médias relativas a um número de 400 amostras.

1/ Valores entre parêntesis são coeficiente de variação e desvio-padrão em porcentagens, respectivamente.

Quadro 3 - Valores determinados para os parâmetros de retratibilidade e densidade básica da madeira de cinco árvores de Eucalyptus saligna

Table $\mathbf{3}$ - Values determined for parameters shrinkage and specific gravity of wood of five Eucalyptus saligna trees

\begin{tabular}{|l|c|c|c|c|c|}
\hline \multirow{2}{*}{ Estatística Descritiva } & \multicolumn{5}{|c|}{ Propriedade Física } \\
\cline { 2 - 6 } & \multicolumn{3}{|c|}{ Contração (\%) $)^{\frac{1}{\prime}}$} & Relação T/R & $\begin{array}{c}\text { Densidade Básica } \\
\left(\mathrm{g} / \mathrm{cm}^{3}\right)\end{array}$ \\
\cline { 2 - 6 } & $\mathrm{V}$ & $\mathrm{T}$ & 7,67 & 1,99 & 0,47 \\
\hline Valor médio & 26,00 & 14,83 & 2,35 & 0,36 & 0,07 \\
Desvio-padrão & 6,89 & 3,85 & 30,69 & 18,12 & 15,64 \\
Coeficiente de Variação (\%) & 26,52 & 25,94 & 10,70 & 2,66 & 0,33 \\
Amplitude de Variação & 34,08 & 19,09 & 340 & 340 & 360 \\
Número de Observações & 362 & 340 & & $\mathrm{R}$ & \\
\hline
\end{tabular}

${ }^{1 /} \mathrm{V}=$ volumétrica, $\mathrm{T}=$ tangencial e $\mathrm{R}=$ radial.

A melhor visualização da variação do teor de equilíbrio higroscópico em função da umidade relativa do ar é apresentada no gráfico da Figura 1.

Diante do bom ajuste dos dados através da análise de regressão, pode-se estimar, com grande precisão, o teor de equilíbrio higroscópico para a madeira de E. saligna, dentro da faixa de 20 até $100 \%$ de umidade relativa. As informações contidas na Figura 1 são relevantes para os procedimentos de secagem da madeira. A equação apresentada poderá subsidiar os sistemas controladores do processo de secagem, proporcionando condições de umidade relativa adequadas, para que seja obtido um determinado valor de teor de umidade na secagem das peças.

O Quadro 3 mostra que a madeira de Eucalyptus saligna caracteriza-se por apresentar um valor de contração elevado, quando comparado ao de outras espécies de mesma faixa de densidade básica. Segundo Mainieri \& Chimelo (1989), o mogno (Swietenia macrophylla) é um exemplo de madeira de elevada estabilidade

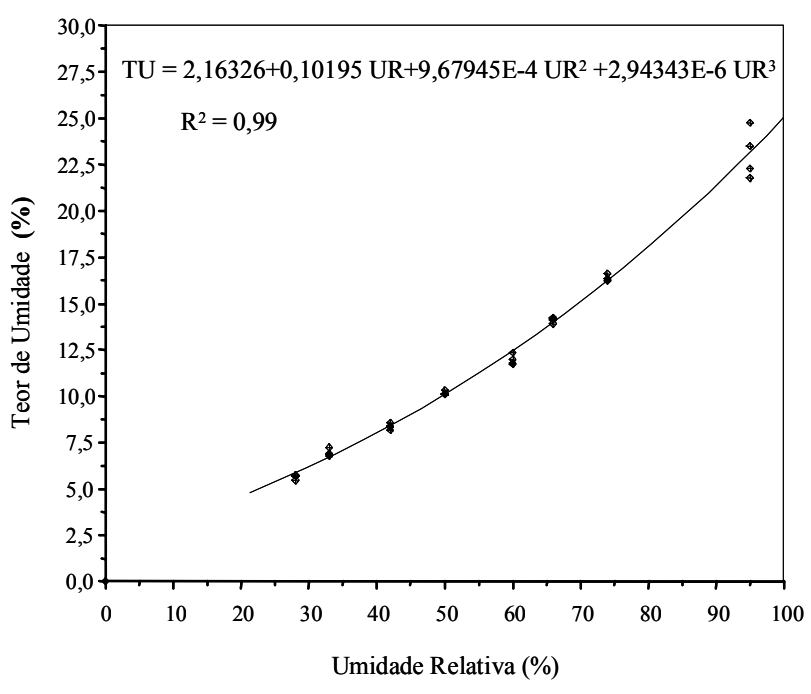

Figura 1 - Representação gráfica do teor de umidade da madeira (TU) para diferentes condições de umidade relativa do ar, à temperatura de $27^{\circ} \mathrm{C}$.

Figure 1 - Moisture content of wood under different conditions of relative humidity of the air, at $27^{\circ} \mathrm{C}$. 
dimensional, mesmo com uma densidade próxima daquela encontrada para a madeira de eucalipto em estudo. Seus valores de contração volumétrica, tangencial e radial são de $8,6,4,5$ e 3,2\%, respectivamente, e a relação $T / R$ é igual a 1,41 , o que a credencia como uma das madeiras mais estáveis dimensionalmente. Quanto ao eucalipto, observa-se uma grande variação na sua retratibilidade, refletida nos elevados valores de coeficiente de variação, no desvio-padrão e na amplitude de variação. Tamanha heterogeneidade poderá refletir de imediato na utilização dessa madeira para aplicações que irão exigir elevada estabilidade dimensional. Apesar de os coeficientes de contração serem elevados, a relação $\mathrm{T} / \mathrm{R}$ mostrou-se muito próxima dos valores encontrados nas madeiras comerciais brasileiras. Segundo informações contidas no IPT (1956), os coeficientes de contração de tais madeiras variam de 3,5 a $8,6 \%$ na direção radial, de 7,8 a $21,9 \%$ na direção tangencial e de 13,2 a $35,7 \%$ na contração volumétrica total. Oliveira (1998), estudando madeira de sete espécies de eucalipto, com 16 anos de idade, encontrou valores de coeficientes de contração volumétrica variando de 15,9 até $27,2 \%$, bem como valores de $T / R$ variando de 1,4 até 2,1. Durlo \& Marchiori (1992) classificaram valores de T/R de 1,5 a 2,0 como sendo normais para madeiras de ipê, pinus, pinheiro-do-paraná, peroba-rosa, teca, entre outras espécies. Carmo (1996), estudando o comportamento da madeira de E. saligna,

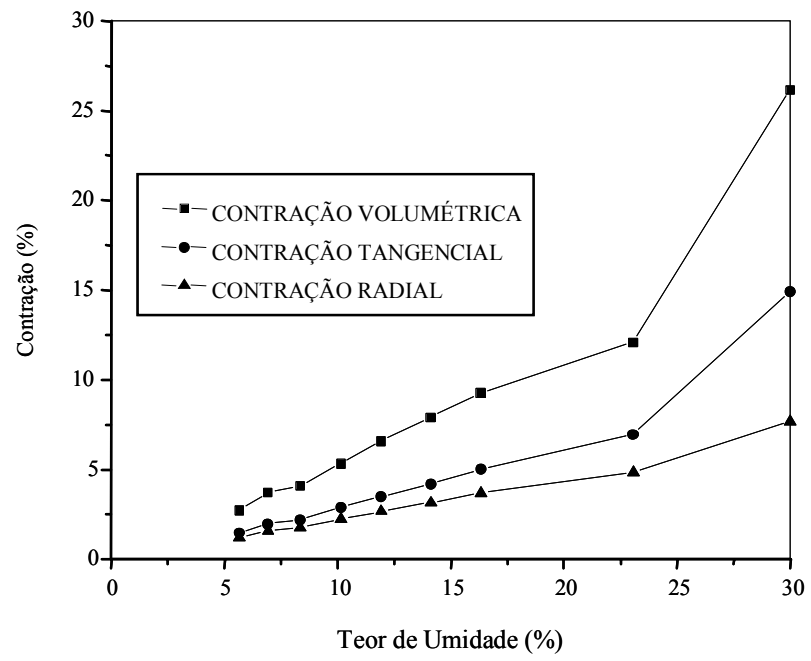

Figura 2 - Representação gráfica das contrações volumétrica e lineares para várias condições de umidades da madeira até à condição anidra, para Eucalyptus saligna.

Figure 2 -Transverse and volumetric shrinkage for different conditions of wood moisture up to an anhydrous condition, for Eucalyptus saligna. com 21 anos de idade, encontrou valores de 23,5, 16,9 e $9,7 \%$, respectivamente, para as contrações volumétrica, tangencial e radial, proporcionando um fator anisotrópico igual a 1,74 .

Uma visualização do comportamento das contrações volumétrica e linear, partindo de diferentes condições de umidade da madeira até a condição anidra, é apresentada no gráfico da Figura 2.

$\mathrm{Na}$ Figura 2, verificam-se elevados coeficientes de contração total, quando a madeira perde umidade do ponto de saturação das fibras (psf) até a condição anidra. Para valores correspondentes a umidades inferiores ao psf, entretanto, os valores totais das contrações diminuem drasticamente à medida que o teor de umidade da madeira diminui. As informações contidas nessa figura são importantes sob o ponto de vista prático, para subsidiar as operações de secagem dessa madeira. Recomenda-se cautela quando a madeira atingir o estágio inicial de secagem abaixo do psf, até valores pouco abaixo dos $25 \%$ de teor de umidade, em que os coeficientes de contração são elevados; à medida que a secagem prossegue, verifica-se redução acentuada nos níveis de contração. Este comportamento das contrações abaixo do psf pode ser mais bem visualizado na Figura 3, onde se verificam contrações mais suaves nas umidades situadas abaixo de $17 \%$.

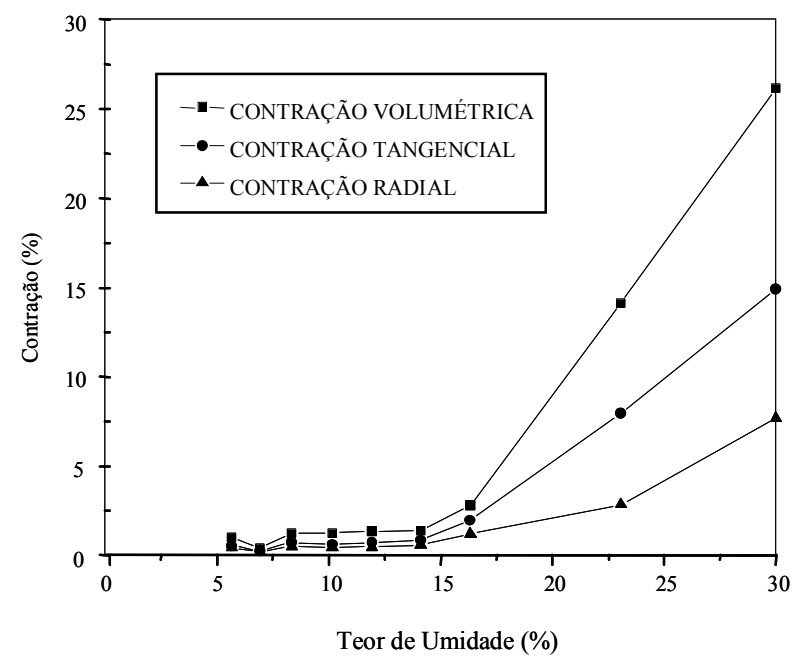

Figura 3 - Representação gráfica da variação das contrações volumétrica e lineares da madeira de Eucalyptus saligna, à temperatura de $27^{\circ} \mathrm{C}$.

Figure 3 - Variation of the transverse and volumetric shrinkage of Eucalyptus saligna wood, at $27^{\circ} \mathrm{C}$.

R. Árvore, Viçosa-MG, v.27, n.2, p.233-239, 2003 


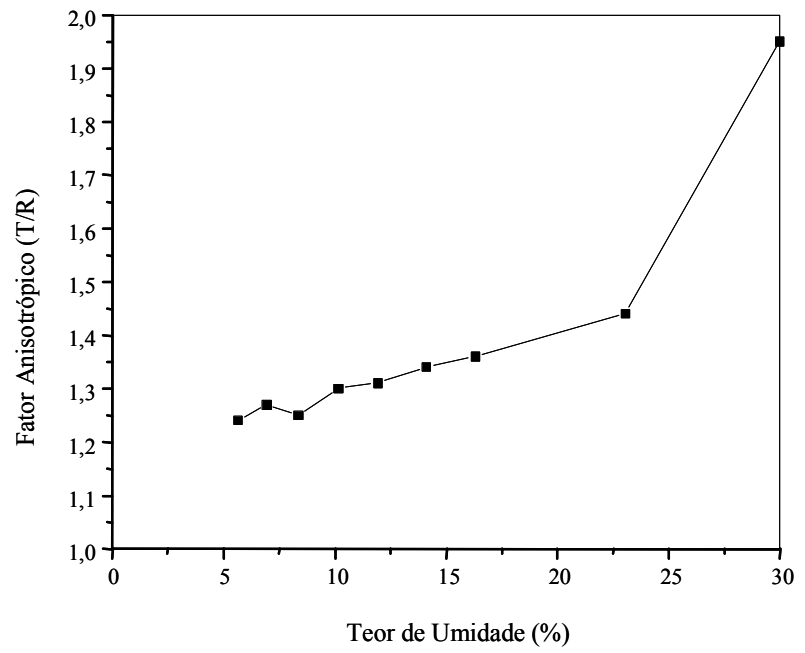

Figura 4 - Representação gráfica do fator anisotrópico (T/R) para secagem da madeira de Eucalyptus saligna de diferentes condições de umidade até a condição anidra, à temperatura de $27^{\circ} \mathrm{C}$.

Figure 4-Anisotropic factor $(T / R)$ for Eucalyptus saligna wood drying under different conditions of moisture up to an anhydrous condition, at $27^{\circ} \mathrm{C}$.

Para teores de umidade inferiores a $20 \%$, verificase que as variações nas contrações foram muito pequenas, o que indica a quase inexistência de problemas para a futura utilização dessa madeira. Recomendam-se, no entanto, condições mais suaves no estágio inicial de secagem, quando a madeira apresenta umidade abaixo do psf. Esta condição mais suave de secagem também é justificada para liberação da água livre da madeira, ou acima do ponto de saturação das fibras. Nesse estágio de secagem verificam-se problemas de colapso. Segundo Cavalcante (1991), a madeira de E. saligna é mais propensa ao colapso que a madeira de outras espécies do mesmo gênero.

O comportamento dessa madeira, no que diz respeito à sua variação dimensional, também pode ser mais bem elucidado pela visualização da alteração do fator anisotrópico, a partir das diferentes condições de teor de umidade da madeira abaixo do psf, como ilustrado na Figura 4.

$\mathrm{Na}$ Figura 4, observa-se uma queda drástica no valor do fator anisotrópico quando a secagem desta madeira decresce a um ponto abaixo do psf. A partir de teores de umidade próximos de $25 \%$, a secagem até a condição anidra faz com que a madeira se torne muito estável. Os

R. Árvore, Viçosa-MG, v.27, n.2, p.233-239, 2003

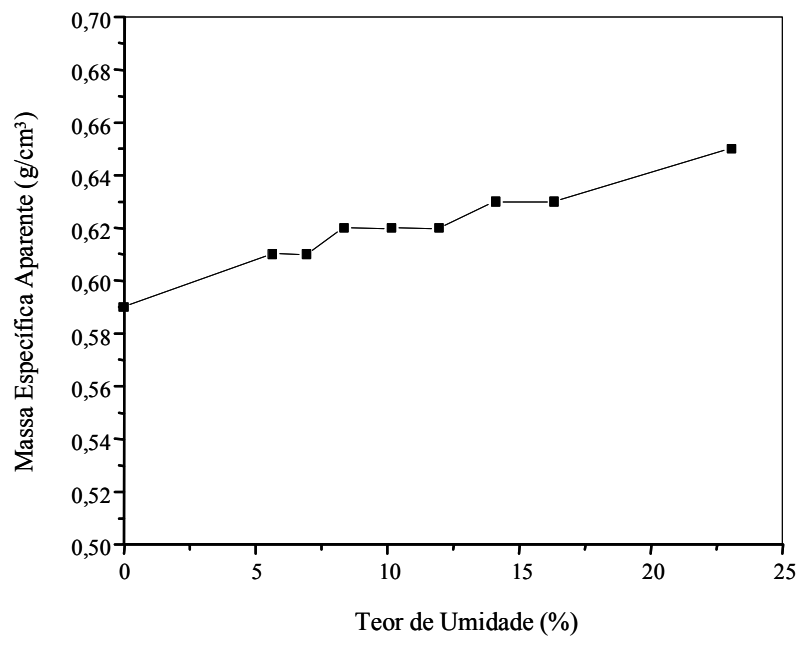

Figura 5 - Representação gráfica da variação da massa específica aparente da madeira de Eucalyptus saligna em diferentes teores de umidade.

Figure 5 - Variation of the apparent density of Eucalyptus saligna wood at different moisture contents.

valores encontrados são, até mesmo, inferiores aos valores encontrados nas madeiras consideradas mais estáveis dimensionalmente.

A Figura 5 apresenta graficamente a variação da massa específica aparente, em função do teor de umidade para a madeira em estudo.

A figura anterior demonstra que, para maiores umidades de equilíbrio da madeira, maiores são os valores de sua massa específica aparente. Esse comportamento poderá ser explicado pela inexistência de proporcionalidade entre a massa perdida ou adicionada à madeira e a sua respectiva alteração de volume.

\section{CONCLUSÕES}

Através dos dados apresentados, conclui-se para a madeira de Eucalyptus saligna em questão que:

- Mesmo para um grande número de amostras secas em diferentes teores de umidades de equilíbrio, o valor médio final de umidade alcançado para a mesma condição de umidade é muito próximo do valor encontrado para uma amostra individual. Tal comportamento é confirmado pelos valores encontrados para o coeficiente de variação e o desvio-padrão. 
- Quanto aos parâmetros relativos à retratibilidade, a madeira caracteriza-se por apresentar valores bastante elevados, se comparados aos das outras madeiras de mesma faixa de densidade.

- Além de elevados coeficientes de contração, a madeira apresentou comportamento bastante heterogêneo, no que diz respeito aos seus coeficientes de contrações, destacado pelos elevados valores de coeficiente de variação e de desvio-padrão.

- Apesar dos elevados coeficientes de contração, o fator anisotrópico ou relação $\mathrm{T} / \mathrm{R}$ manteve-se próximo daquele encontrado pela grande maioria das madeiras comerciais brasileiras.

- Não existe proporcionalidade entre os coeficientes de contração na variação higroscópica abaixo do ponto de saturação das fibras.

\section{AGRADECIMENTO}

Os autores agradecem ao Dr. José Carlos Duarte Pereira e ao Centro Nacional de Pesquisas Florestais da EMBRAPA - Colombo-PR, pela cessão da madeira utilizada e pelas informações dispensadas para a realização desse trabalho.

\section{REFERÊNCIAS BIBLIOGRÁFICAS}

BARROSO, A. B. Silvicultura especial de árboles maderables tropicales. Cuba: Editorial Técnica Científico, 1983. $427 \mathrm{p}$.

BOLAND, D. J. et al. Forest trees of Australia. Adelaide: CSIRO, 1984. 687p.

CARMO, A. P. T. Avaliação de algumas propriedades da madeira de seis espécies de eucalipto. 1996. $74 \mathrm{f}$.

Dissertação (Mestrado em Ciência Florestal) -

Universidade Federal de Viçosa, Viçosa-MG, 1996.

CAVALCANTE, A. A. Ocorrência de colapso na secagem da madeira de Eucalyptus grandis e Eucalyptus saligna.

1991. 76p. Dissertação (Mestrado em Ciências Florestais) Escola Superior de Agricultura "Luiz de Queiroz",

Piracicaba, 1991. p
DURLO, M. A.; MARCHIORI, J. N. C. Tecnologia da madeira: Retratibilidade Santa Maria, CEPEF/FATEC, 1992. 33p. (Série Técnica, 10)

GÁL, S. Die methodic der wasserdampfsorptionsmessungen. Berlin: Springer-Verlag, 1957. 242 p.

HILLIS, W.E.; BROWN, A.G. (Eds.) Eucalypts for wood production. Melbourne: CSIRO, 1978. $434 \mathrm{p}$.

INSTITUTO DE PESQUISAS TECNOLÓGICAS - IPT. Métodos de ensaios adotados no IPT para o estudo de madeiras nacionais: tabelas de resultados obtidos para madeiras nacionais, nomenclatura das madeiras nacionais. 2.ed. São Paulo: 1956. 62 p. (Boletim Técnico, 31)

KOLLMANN, F. F. P.; COTÊ, W. A. Principles of wood science and technology. New York: Springer-Verlag, 1968. v. 1.

LIMA, T. G. Variações no sentido radial e longitudinal de algumas propriedades das madeiras de Eucalyptus microcorys F. Muell e Eucalyptus pilularis Sm. 1996. 106 f. Dissertação (Mestrado em Ciência Florestal) Universidade Federal de Viçosa, Viçosa-MG, 1996.

MAINIERI, C.; CHIMELO, J.P. Fichas de características das madeiras brasileiras. 2.ed. São Paulo: IPT, 1989. 418 p. (Publ. IPT, 1791)

MASSERANN, C.; MARIAUX, A. Anisotropic de retrait et estructure du bois. Recherche de l'influence des caracteres morphologiques transverses des fibres. Bois et Forêts des Tropiques, n. 209, p. 35-47, 1985.

OLIVEIRA, J.T.S. Caracterização da madeira de eucalipto para a construção civil. 1998. $429 \mathrm{f}$. Tese (Doutorado) - Universidade de São Paulo, São Paulo, 1998.

PANSHIN, A. J.; DE ZEEUW, C. Textbook of wood technology. 4.ed. New York: Mc-Graw-Hill, 1980. 722 p.

TSOUMIS, G. Science and technology of wood: structure, properties, utilization. New York: Van Nostrand Reinhold, 1991. 494 p. 